\title{
Spatial-Frequency Approach to Fibrous Tissue Classification in Intracoronary Optical Images
}

\author{
Maysa MG Macedo ${ }^{1}$, Pedro FG Nicz², Carlos M Campos², Pedro A Lemos ${ }^{2}$, Marco A Gutierrez ${ }^{1}$ \\ ${ }^{1}$ Division of Informatics, Heart Institute, University of Sao Paulo Medical School, Brazil \\ ${ }^{2}$ Department of Hemodynamics, Heart Institute, University of Sao Paulo Medical School, Brazil
}

\begin{abstract}
Increased understanding about the mechanisms of coronary thrombosis in humans has been limited by the lack of imaging modalities with resolution sufficient to characterize fibrous cap tissue and determine its thickness in vivo. Intravascular optical coherence tomography (IOCT) provides images with micrometer axial $(10-15 \mu \mathrm{m})$ and lateral resolution $(40 \mu \mathrm{m})$, enabling detailed visualization of micro-structural changes of the arterial wall. This article describes a fully automated method for identification and quantification of fibrous tissue in IOCT human coronary images based on spatial-frequency analysis by means Short-Time Fourier transform. Forty IOCT frames from nine IOCT in-vivo datasets were annotated by an expert and used to evaluate the proposed fibrous tissue characterization method.
\end{abstract}

\section{Introduction}

The most frequent cause of coronary occlusion is rupture of thin-cap fibroateroma (TCFA) plaques [1]. Plaque rupture with subsequent thrombosis is the most frequent cause of acute coronary syndrome. Usually these lesions are characterized by a large necrotic core with a fibrous cap of thickness less than $65 \mu \mathrm{m}$, and a plaque rupture occurs when the cap thickness is $23 \pm 19 \mu m$ [2].

Kume et al. [3] demonstrated that recent technological advances in intravascular imaging technology such as optical coherence tomography (IOCT) provides an accurate representation of the thickness of the fibrous cap and an useful tool to assess the vulnerability in lipid-rich plaques. Frameworks have been developed using IOCT as a modality that aim to quantify the thickness of the fibrous cap using (i) the attenuation coefficient based on image intensity [4] and; (ii) a semi-automatic method to quantify TCFA by dynamic programming [5].

Usually fibrous tissue has a texture in IOCT images that is more homogeneous and with higher backscattering than all other areas in the image. However, changes may occur in brightness according to the position of the catheter at the time of slice acquisition. Methods based only on texture analysis [6] and intensity of the pixels may be adversely affected by changes in light intensity caused by the catheter movement. Thus, frequency domain-based methods can be an alternative to overcome this image intensity problems.

If the spectrum is calculated for an image that contains several different types of tissue, it is possible to find the spectral component with the highest amplitude without localization of the frequency in the spatial domain. A solution to identify more than one tissue is to calculate the spectrum for each window of the image where the accuracy of this spectrum is directly proportional to the size of this window.

There are several methods of frequency analysis relative to a consecutive unit of measurement (eg. time, distance) amongst them, we can highlight: Short-time Fourier transform (STFT), Wigner-Ville distribution (WVD) and ChoiWilliams distribution (CWD) [7]. But the objective is the same: analyze spectrum for each time or distance interval. Thus, we have developed a fibrous tissue identification method based on space-frequency methods that is less sensible to changes in lighting than methods restricted to space analysis. We can calculate the spectrum of a given tissue and analyze which frequencies are significant.

In this paper we propose STFT-based method to identify fibrous tissue in IOCT images, an optimization method to select the best features and a validation of the results comparing with manual segmentations, achiving $80 \%$ and $98.6 \%$ of sensitivity and specificity, respectively.

\section{Materials and Methods}

Given an IOCT frame, the proposed method to identify and measure the thickness of the fibrous tissue consists of the following 5 steps: (i) identification of the lumen border; (ii) calculation of the spectrum for each different depth by means of STFT; (iii) spectral analysis; (iv) fibrous tissue identification; (v) fibrous tissue thickness quantification. 


\subsection{Image data}

Images were acquired using a Fourier-Domain OCT (FD-OCT) system (C7-XR - OCT Intravascular Imaging System, St. Jude Medical, St. Paul, Minnesota) at the Heart Institute, University of Sao Paulo Medical School (INCOR-HC FMUSP), Brazil. The study protocol was approved by the institutional review board (CAPPesq) under register no. 0243/08. The system was equipped with a Dragonfly catheter with a diameter of $0.90 \mathrm{~mm}$, a Guide Wire (GW) with a maximum outer diameter of $0.3556 \mathrm{~mm}$ (ImagewireTM, LightLab Imaging), and working length of $135 \mathrm{~cm}$. Pullback speed was $20 \mathrm{~mm} / \mathrm{sec}$ over a distance of $54.0 \mathrm{~mm}$, totalizing 271 frames. The $2 \mathrm{D}$ input images to the proposed method were in DICOM format with dimensions $1024 \times 1024$ pixels and spatial resolution of $10 \times 10 \mu \mathrm{m}$ in Cartesian coordinates. In total, $402 \mathrm{D}$ images from 9 patients were used, 6 IOCT frames for the training phase and 34 for testing.

\subsection{Lumen border detection}

The input images were transformed to polar coordinates, and the lumen detected by the method described in [8], based on a bilateral filter and morphological operations. The lumen border indicates where the vessel wall starts, limiting the input signal. Each A-line in the polar image is an intensity profile where each points is related to a different depth, as shown in Figure 1, and is used as an input signal for the next stage of the processing.
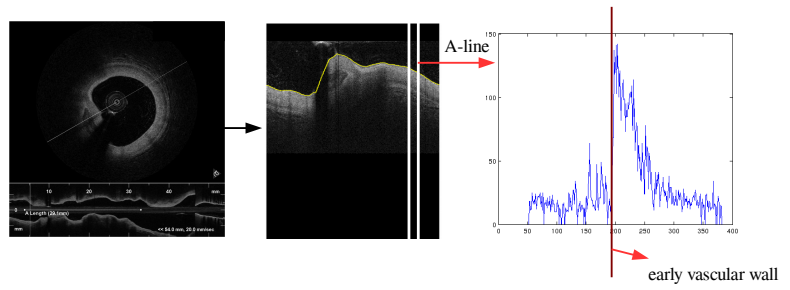

Figure 1. The Cartesian image is transformed to a polar coordinates and each A-line (column) provides an intensity profile. The lumen border detection defines where the vessel wall starts.

\subsection{Short-time Fourier transform}

This method consists of multiplying a signal by a window function $h$ with a given length $\delta$ to produce a modified signal, as shown in Equation 1, and perform the Fourier transform in this signal.

$$
s^{\prime}(\delta)=s(\delta) h(\delta-d)
$$

The STFT is calculated for each A-line (input signal) in the polar image, where each points is related to a different depth $d$, and it is represented by Equation 2, resulting in a three-dimensional information: depth, frequency and magnitude. The Hanning function was used as window function $h$. Figure 2 shows the input signal $s(d)$ multiplied by the window function $h(\delta-d)$ followed by calculation of the FFT in different depths $d$. The result is a frequency spectrum for each $d$, as illustrated in Figure 3 a).

$$
\operatorname{STFT}(\omega, d)=\int s(\delta) h(\delta-d) e^{-j \omega \delta} d \delta
$$

where $\omega$ is the angular frequency.

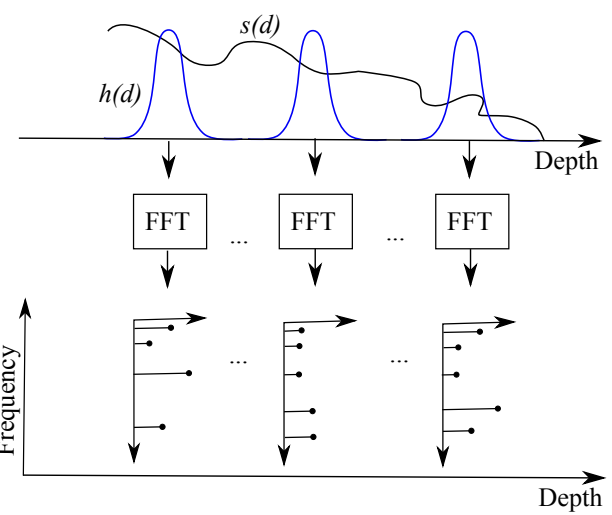

Figure 2. STFT scheme adapted from Quian et al. . The intensity value in an IOCT A-line scan is presented as the function $s(d)$, which is windowed by the function $h(d)$.

\subsection{Spectral analysis}

Information was extracted from each spectrum calculated along the A-line, such as:

- Maxmag (maximum magnitude ):

$$
\operatorname{Maxmag}(d, \Omega)=\max \{M(d, \Omega)\}
$$

- Meanmag (mean magnitude):

$$
\operatorname{Meanmag}(d, \Omega)=\overline{M(d, \Omega)}
$$

- $F_{\max }$ (frequency of the maximum magnitude):

$$
\left\{F_{\max } \mid M\left(d, F_{\max }\right)=\max \{M(d, \Omega)\}\right\}
$$

- $F_{\text {mean }}$ (mean frequency):

$$
F_{\text {mean }}(d, \Omega)=\sum_{i=1}^{n} \omega_{i} * P\left(d, \omega_{i}\right)
$$

- Fmagmean (mean of frequency multiplied by magnitude ):

$$
\operatorname{Fmag}_{\text {mean }}(d, \Omega)=\overline{\Omega * M(d, \Omega)}
$$

- STDF (standard deviation of frequency):

$$
\operatorname{STDF}(d, \Omega)=\sum_{i=1}^{n}\left(\omega_{i}-F_{\text {mean }}\right)^{2} * P\left(d, \omega_{i}\right)
$$


- E (energy density spectrum):

$$
E(d, \Omega)=\sum_{i=1}^{n} P\left(d, \omega_{i}\right)
$$

where $\omega$ is the angular frequency, $\Omega=\left\{\omega_{1}, . ., \omega_{n}\right\}$, and $M(d, \Omega)=|\operatorname{STFT}(\Delta d, \Omega)|$ and $P(d, \Omega)=$ $|\operatorname{STFT}(\Delta d, \Omega)|^{2}$ are the magnitude and the power in the depth $d$ and for frequencies $\Omega$, respectively.

These measures are calculated based on a depth interval $\Delta d=[d-\delta, . ., d+\delta]$ as shown in Figure $3 \mathrm{~b}$ ) where the region bounded by a rectangle has the values considered to the computing for each $d$. The window lengths, $\delta$, were tested with the following values: 16,32 and 64 pixels, with $\delta=64$ pixels providing the optimal result.

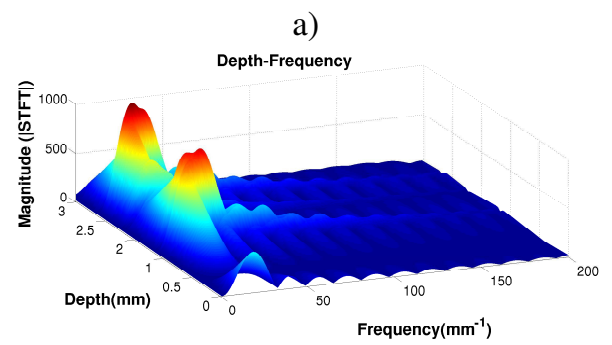

b)

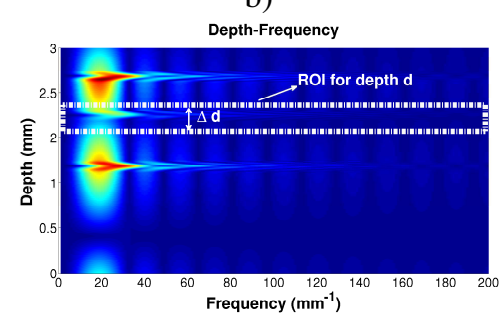

Figure 3. Depth-frequency plot: a) 3D surface plot showing variation of magnitude of the STFT with changing depth and frequency; b) a 2D plot, showing the same profile as in a), with a hashed rectangle is indicating the neighborhood for spectral metrics related to the depth $d$.

\subsection{Fibrous tissue identification}

The identification of fibrous tissue is made by combining the metrics described above. The best parameters were chosen by the optimization method Forward Regression Orthogonalization Least Squares (FROLS) [9], which found a set of best features and its weights for a linear combination. If the value of linear combination for each pixel is $\geq 0.5$, considering the range $[0,1]$, the pixel is labeled as fiber. This optimization method used 6 frames labeled by a specialist for the training phase. The best measures determined by FROLS were: the maximum magnitude, the mean of frequency multiply by magnitude and energy density spectrum.

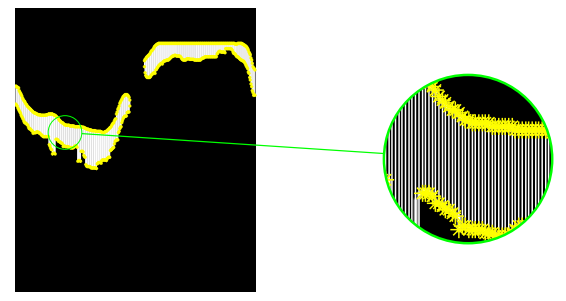

Figure 4. IOCT frame in polar coordinates showing automated identification of fibrous tissue (yellow) and distance between inner and outer contour (white).

\subsection{Thickness quantification}

In the IOCT images, the fibrous tissue thickness is defined as the distance from the internal border of the signalrich layer, nearest the lumen, to the internal border of the signal-poor middle layer or to the internal border of the signal-poor region in the case of lipid pools or calcium plaques. The distance is computed from the polar image in increments of 1 degree. Figure 4 shows the distance in white color.

\subsection{Validation}

The evaluation of the fibrous tissue segmentation method was based on manual segmentation by an expert. A set of seven metrics was used to measure the accuracy of the proposed automated method for fibrous tissue identification. These metrics are: Mean absolute difference of area (MADA), Hausdorff distance (H), RMS Symmetric surface distance (RMSSSD), Specificity(SPE), Sensitivity (S), Accuracy (ACC), Dice metric (DM). More details about the metrics are presented in [8].

\section{Results}

Tests were carried out on a total of 34 images with atherosclerotic plaque obtained during 7 acquisitions. The fibrous area was obtained without any user interaction. The mean difference between the automated and the manual fiber tissue area was found to be $1.1 \mathrm{~mm}^{2}$. Considering the overlap of both results, the automated method achieved $80.0 \%$ and $98.6 \%$ for sensitivity and specificity, respectively. Table 1 shows all evaluation metrics of the proposed automated method. Figure 3 shows the results of automated fibrous tissue identification compared with manual segmentation.

\section{Discussion and Conclusion}

A limitation of the frequency analysis in this study comes from choosing a small window to optimize spatial resolution and select pixels within a single region, which 
Table 1. Evaluation of automated fibrous tissue identification for 34 frames.

\begin{tabular}{ccccccc}
\hline $\begin{array}{c}\text { RMSSSD } \\
(\mathrm{mm})\end{array}$ & $\begin{array}{c}\text { DICE } \\
(\%)\end{array}$ & $\begin{array}{c}\text { MADA } \\
\left(\mathrm{mm}^{2}\right)\end{array}$ & $\begin{array}{c}\text { S } \\
(\%)\end{array}$ & $\begin{array}{c}\text { SPE } \\
(\%)\end{array}$ & $\begin{array}{c}\text { ACC } \\
(\%)\end{array}$ & $\begin{array}{c}\text { H } \\
(\mathrm{mm})\end{array}$ \\
\hline 0.13 & 76.3 & 1.1 & 79.9 & 98.6 & 97.4 & 0.40 \\
\hline
\end{tabular}
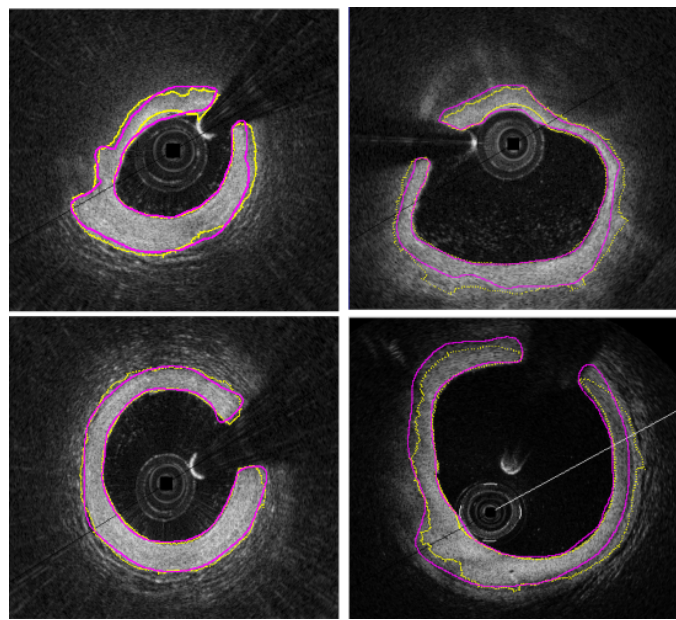

Figure 5. IOCT images segmented by the proposed method. The fiber tissue areas are identified using the automated method (yellow) and the manual segmentation (magenta).

limits the number of frequency bins for the Fourier transform.

Ughi et al. [6] developed an automated tissue characterization based on texture analysis, attenuation coefficient and pixel classification using Random Forest, reaching an accuracy of $89.5 \%$ for fibrous tissue, which is lower than our method (97.4\%). Athanasiou et al. [10] developed an automated method based on segmentation and classification using K-means and achieved $87 \%$ and $0.09 \mathrm{~mm}^{2}$ for sensitivity and MADA respectively, whereas we obtained $80 \%$ and $1.1 \mathrm{~mm}^{2}$ for these measures. Despite not having a better result comparing with [10], we believe these results can be improved with the incorporation of the attenuation coefficients into the methodology proposed in [11].

\section{Acknowledgements}

The authors thank Sao Paulo Research Foundation (FAPESP) for their financial support (grant \#2013/09922-8) and NAP eScience-USP for use of their equipment.

\section{References}

[1] Mukherjee D, Bates E, Roffi M, Moliterno D. Cardiovascular Catheterization and Intervention: A Textbook of Coro- nary, Peripheral, and Structural Heart Disease. 2010.

[2] Virmani R, Burke AP, Farb A, Kolodgie FD. Pathology of the vulnerable plaque. Journal of the American College of Cardiology 2006;47(8 Suppl):C13-8. ISSN 1558-3597.

[3] Kume T, Akasaka T, Kawamoto T, Okura H, Watanabe N, Toyota E, Neishi Y, Sukmawan R, Sadahira Y, Yoshida K. Measurement of the thickness of the fibrous cap by optical coherence tomography. American Heart Journal October 2006;152(4):755.e1-4. ISSN 1097-6744.

[4] van Soest G, Goderie T, Regar E, Koljenović S, van Leenders GLJH, Gonzalo N, van Noorden S, Okamura T, Bouma BE, Tearney GJ, Oosterhuis JW, Serruys PW, van der Steen AFW. Atherosclerotic tissue characterization in vivo by optical coherence tomography attenuation imaging. Journal of Biomedical Optics 2010;15(1):011105. ISSN 1560-2281.

[5] Wang Z, Chamie D, Bezerra HG, Yamamoto H, Kanovsky J, Wilson DL, Costa MA, Rollins AM. Volumetric quantification of fibrous caps using intravascular optical coherence tomography. Biomedical Optics Express June 2012; 3(6):1413-26. ISSN 2156-7085.

[6] Ughi GJ, Adriaenssens T, Sinnaeve P, Desmet W, D’hooge J. Automated tissue characterization of in vivo atherosclerotic plaques by intravascular optical coherence tomography images. Biomedical Optics Express July 2013; 4(7):1014-30. ISSN 2156-7085.

[7] Qian S. Joint Time-frequency Analysis: Methods and Applications. Illustrated edition. PTR Prentice Hall, 1996.

[8] Macedo MMG, Guimarães WVN, Galon MZ, Takimura CK, Lemos PA, Gutierrez MA. A bifurcation identifier for IV-OCT using orthogonal least squares and supervised machine learning. Computerized Medical Imaging and Graphics September 2015;ISSN 1879-0771.

[9] Billings SA. Nonlinear System Identification: NARMAX Methods in the Time, Frequency, and Spatio-Temporal Domains. 2013. ISBN 978-1-119-94359-4.

[10] Athanasiou LS, Bourantas CV, Rigas G, Sakellarios AI, Exarchos TP, Siogkas PK, Ricciardi A, Naka KK, Papafaklis MI, Michalis LK, Prati F, Fotiadis DI. Methodology for fully automated segmentation and plaque characterization in intracoronary optical coherence tomography images. Journal of Biomedical Optics 2014;19(2):026009. ISSN 1560-2281.

[11] Vermeer Ka, Mo J, Weda JJa, Lemij HG, de Boer JF. Depthresolved model-based reconstruction of attenuation coefficients in optical coherence tomography. Biomedical optics express December 2013;5(1):322-37. ISSN 2156-7085.

Address for correspondence:

Maysa Malfiza Garcia de Macedo

Servico de Informatica - InCor Av. Dr. Eneas de Carvalho Aguiar, 44 - 05403-900 - Sao Paulo-SP- Brazil

maysa@ime.usp.br 